\title{
Emerging Flux and the Heating of Coronal Loops
}

\author{
B. Schmieder ${ }^{1,2}$, P. Démoulin ${ }^{1}$, D.M. Rust ${ }^{3}$, M.K. Georgoulis ${ }^{3}$, \\ and P.N. Bernasconi ${ }^{3}$ \\ 1. Observatoire de Paris, LESIA, 92195 Meudon Cedex, France \\ 2. ITA, University of Oslo, Blindern, N-0315, Oslo, Norway \\ 3. JHU/APL, 11100 John Hopkins Road, Laurel MD 20723-6099, USA
}

\begin{abstract}
Observations of various instruments on board Yohkoh, SOHO, and TRACE complement high-resolution observations of the balloonborne Flare Genesis Experiment, obtained on January 25, 2000. A subset of the TRACE loops are located in the vicinity of the SXT loops in the NOAA emerging active region 8844 , but never coincide with them. We find that coronal loops appeared $6 \pm 2 h r$ after the first detection of emerging magnetic flux. The loops evolved rapidly when the active region entered its impulsive flux emergence phase. In the low chromosphere, flux emergence was reflected in intense Ellerman bomb activity. Besides chromosphere, we find that Ellerman bombs may also heat the transition region, by contributing to the moss emission. Areas prolific in Ellerman bombs show moss $\sim 100 \%$ brighter than areas without Ellerman bombs. Only the strongest Ellerman bombs can heat their surroundings to coronal temperatures. In the corona, we find a spatio-temporal anticorrelation between the soft X-ray (SXT) and the extreme ultraviolet (TRACE) loops: First, the SXT loops preceded the appearance of the TRACE loops by $30-40 \mathrm{~min}$. Second, the TRACE loops had different shapes and different footpoints compared to the SXT loops. The SXT and TRACE loops are probably formed independently.
\end{abstract}

\section{Introduction}

As magnetic flux emerges into the corona, bright loops appear at all temperatures, and the loops over emerging flux regions are usually the brightest features in the corona (outside flare times). Many questions arise: How these loops are formed? What is principal mechanism of heating? Is there a unique mechanism that can heat these loops at different temperatures? Is there any link between coronal warm (TRACE) and hot (SXT) loops?

\section{Observations}

The Flare Genesis Experiment (FGE) observed NOAA AR 8844 from 15:50 UT to 19:16 UT on January 25, 2000. An example of the longitudinal magnetic field 

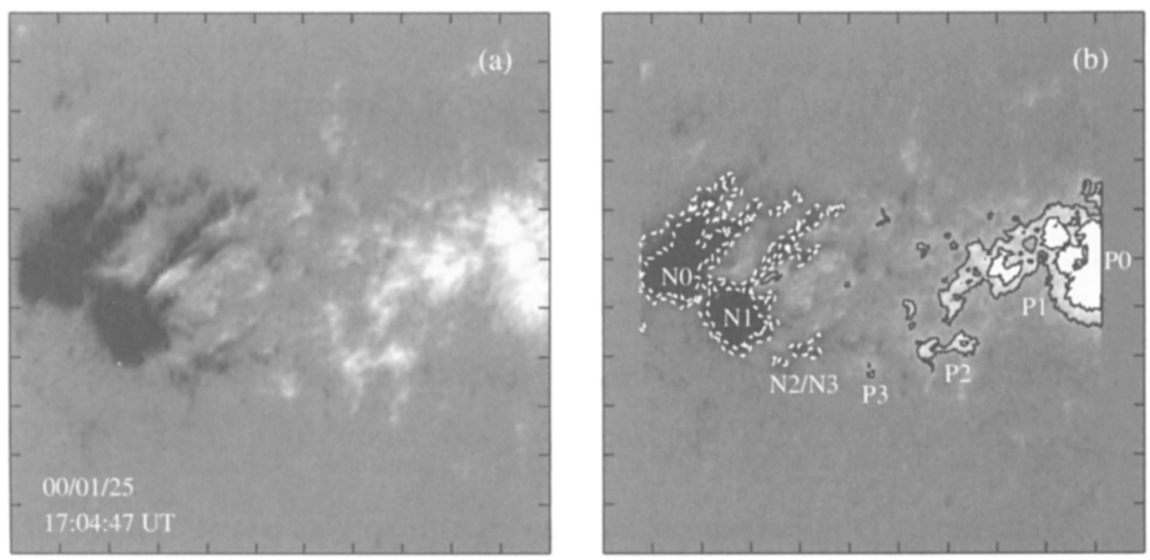

Figure 1. FGE longitudinal magnetogram of AR NOAA 8844 at 17:04:47 UT on January 25, 2000 (left panel without contours, right panel with overlaid contours of \pm 500 and 1000 Gauss). The letters indicate the successive emerging bipoles (N0P0, N1P1, N2P2, N3P3). The solar North direction makes an angle of 27 degrees with the vertical of the figure in a counterclockwise direction. The pixel size is $0 .{ }^{\prime \prime} 18$. The field-of-view is $\sim 92^{\prime \prime} \times 92^{\prime \prime}$. Notice the strong fragmentation of the newly emerging flux in the central part of the active region.

in the region is shown in Figure 1, where we have also indicated the emergence of successive magnetic dipoles. Notice the fragmentation of the flux emergence process, revealed by the FGE observations. The MDI observations permit to set the FGE observations in the context of the long-term (few days) emergence. The first appearance of a new bipole in the quiet part of the Sun occurred on January 23 at 08:03 UT. At the middle of 24 January the bipole increased rapidly in size and strength. On January 25 the FGE observations revealed a hierarchy of parallel bipoles (N0P0, N1P1, N2P2, N3P3). SXT provided both full-disk X-ray images (pixel size $4^{\prime \prime} .92$ ) and partial X-ray images (pixel size $2^{\prime \prime} .46$ ). The SXT filters were sensitive in temperatures $10^{6}-10^{7} \mathrm{~K}$. SXT started observing NOAA AR 8844 on high-resolution mode at 15:11 UT on January 25, with a cadence $\sim 1$ min. TRACE provided observations of the AR in white-light continuum, the low chromosphere $(1600 \AA)$, and the transition region $(171 \AA$ and $195 \AA)$. The cadence for the EUV images at $171 \AA$ and at $195 \AA$ is $80 s$ and the pixel size in the TRACE images is $0^{\prime \prime} .5$.

\section{Relation between EUV and Soft X-Ray loops}

Figure 2 shows an attempt to co-align the TRACE and the SXT images. The coalignment was accomplished in two steps: First, we compared Yohkoh and MDI full-disk images and then we co-aligned MDI and TRACE white-light images. In the upper row of Figure 2 we show three typical TRACE frames, taken at $171 \AA$. The contours of the magnetic field strength and the labels of the various magnetic concentrations are given for reference. We find that two groups of EUV 

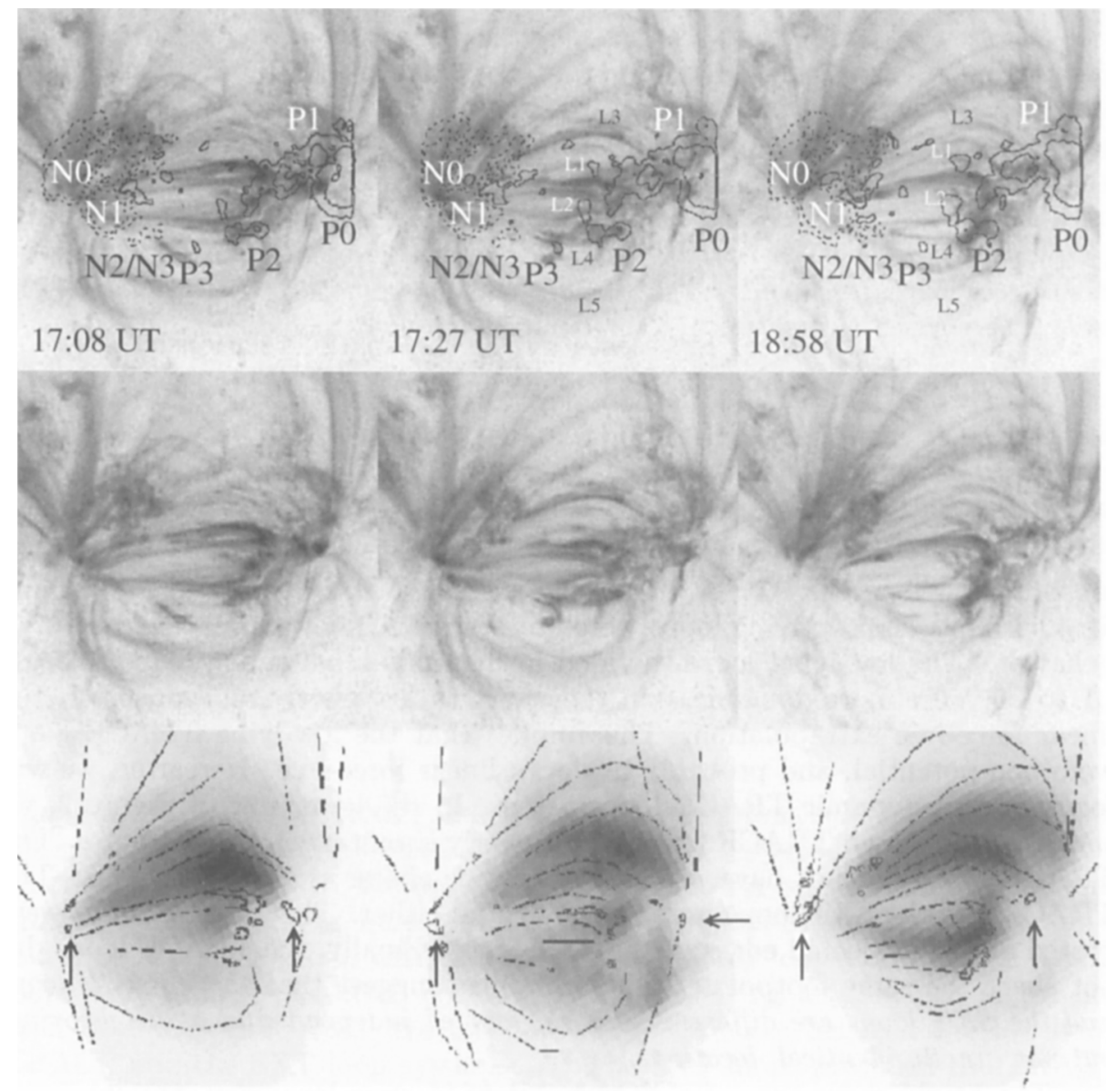

Figure 2. Co-alignment between the TRACE and the SXT loops: Upper row: Negatives of typical TRACE images taken at $171 \AA$. The contours of the magnetic field strength are also shown, taken at $500 \mathrm{G}$ and at $1000 \mathrm{G}$, while the labels of the magnetic polarities are given for reference. Solid/dashed contours indicate positive/negative polarity. The low-lying system of TRACE loops has been classified into five discrete loop sub-systems, labeled L1 to L5. Middle row: The same TRACE images without contours and labels. Lower row: Negatives of nearly simultaneous SXT images (taken within $5 \mathrm{~min}$ from the TRACE observations) co-aligned with the shown TRACE images. The TRACE loops are represented by the dotted curves. The small contours indicated by the arrows correspond to brighter moss features, and they include the calculated locations of the SXT loop footpoints. 

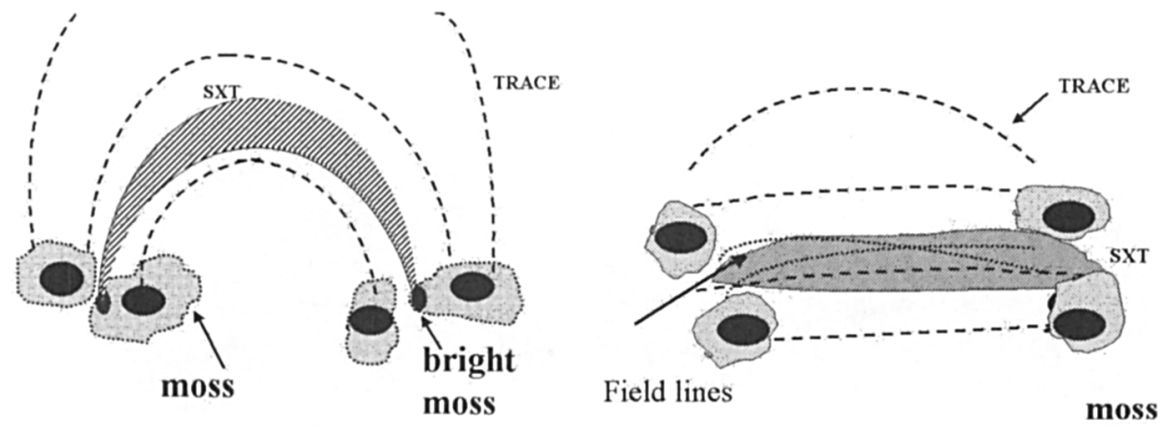

Figure 3. Graphical representation of the spatial relationship between the TRACE and the SXT loops in NOAA AR 8844

loops appeared in the AR: A high-rising system of thin loops and a low-lying loop system. The overlying loops were quiescent and showed a nearly potential behavior. The low-lying loops, divided in five individual sub-systems, labeled L1 to L5, were more dynamic, and they were rather poorly reproduced by the linear force-free extrapolation. This implies that the low-lying TRACE loops were non-potential, and probably not even linear force-free. Hereafter, we will focus on this dynamic TRACE loop system. In the lower row of Figure 2, we show the co-aligned TRACE frames with nearly simultaneous SXT frames. The TRACE loops did not have precisely the same shape as the SXT loops. The TRACE and the SXT loops were not co-spatial either. The TRACE loops were rooted at the projected edges of the SXT loops. Finally, the TRACE loops did not share the same footpoints. These findings suggest that the TRACE loops and the SXT loops are different entities, formed independently at neighboring, but not exactly identical, locations.

The bright moss areas for NOAA AR 8844 were located in the interspot area, roughly overlying the polarities $\mathrm{P} 1$ and $\mathrm{P} 2$ on the west, and occupying the areas just west of the polarities N1 and N2 on the east. The footpoints of the SXT loops roughly corresponded to the brighter moss areas. Therefore, the SXT loops occupied the interspot area in the AR, between P0-P1 and N0-N1 (arrows in Figure 2). From the extrapolation we also find that the separation between the SXT loop footpoints is larger than the separation between the neighboring TRACE loop footpoints. As a result, the SXT loops are longer and higher than their nearby TRACE loops.

Analysing the temporal evolution of the loops is a complementary way to study their relation-ship. In particuliar, a new system of SXT loops appeared in the south after 17:10 UT. Their arrival corresponded to the emergence of the south bipole N2P2 (first seen on the magnetogram at 16:03 UT. The last TRACE images of the first orbit, at 17:27:31 UT, do not show any change in the southern loops, but there are some brightenings that may be new moss corresponding to the emergence of bipole N3-P3 (Fig. 2). We conclude that SXT loops formed between 30 and 50 minutes before TRACE loops. 


\section{Dissipation of Ellerman bomb energy}

FGE observed several hundreds of Ellerman bombs (EBs) at the blue wing of the $H \alpha$ line. These events were studied by Georgoulis et al. (2002) and Bernasconi et al. (2002). They occur and recur in preferential locations. Areas showing enhanced EB triggering are the boundaries of evolving magnetic field concentrations, or colliding magnetic configurations. Georgoulis et al. (2002) studied the statistical properties of EBs and found that EBs may heat the low chromosphere of emerging flux regions. Whether the EBs' heating role is restricted in the low chromosphere is unclear. To investigate the heating role of EBs / bright points (BPs) observed at $1600 \AA$ we focus on the moss brightness of moss in the transition region (Schmieder et al. 2004). Inspecting the average moss intensity, we find that moss areas which almost always included EBs, are consistently bright moss areas. The fainter moss area contained fewer EBs than any other moss area. Therefore, EBs / $1600 \AA$ BPs may heat the transition region and contribute to moss brightness, besides heating the chromosphere of emerging flux regions. This conjecture is not meant to imply that EBs occur only at the footpoints of soft X-ray loops. The presence of EBs in the boxes is not necessary linked to the SXT loops. EBs were linked to the photospheric conditions and occurred in various locations in the AR, independently from the SXT footpoints.

\section{Conclusion}

Simultaneous FGE, MDI, SXT, and TRACE observations enabled us to correlate the spatial locations of the Soft X-ray and the EUV loops in the NOAA emerging flux region 8844. The EUV loops at $171 \AA$ and at $195 \AA$ consisted of two different groups: An over-arching, quiescent group, not matched by the SXT loops, and a low-lying, dynamic group with neighboring soft X-ray loops. The high TRACE loops were almost potential, contrary to the low-lying loops which might not even be linear force-free. We find an anti-coincidence between the low-lying TRACE and the SXT loop systems: (i) The SXT loops preceded the TRACE loops by about $30-40 \mathrm{~min}$. (ii) The SXT loops were higher and longer than their corresponding TRACE loops. (iii) The TRACE loops were located on the projected boundaries of the SXT loops. (iv) The SXT loops were rooted in brighter moss areas. These areas corresponded to relatively strong magnetic fields (several $10^{2} G$ to $10^{3} G$ ) in the interspot area of the AR. The TRACE loops were rooted in areas of relatively weak magnetic fields, not stronger than a few hundred $G$ (Fig. 3).

\section{References}

Bernasconi, P. N., Rust, D., M., Georgoulis, M. K., \& Labonte, B. J. 2002, Solar Phys, 209, 119

Georgoulis, M. K., Rust, D. M., Bernasconi, P. N., \& Schmieder, B. 2002, ApJ, 575,506

Schmieder, B., Georgoulis, M. K., Rust, D. M., Démoulin, P., \& Bernasconi, P. N. 2004, ApJ, in press 